\title{
Aquaporins in peripheral nociception
}

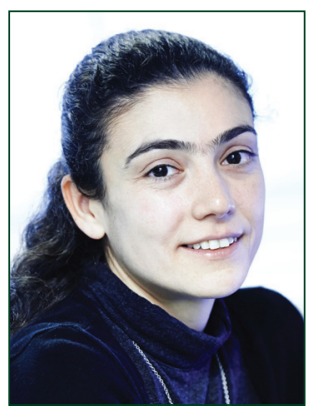

Parisa Gazerani* “...many questions remain unanswered about the roles of aquaporins in the peripheral nervous system and in particular, in peripheral pain signaling."
First draft submitted: 28 November 2016; Accepted for publication: 30 November 2016; Published online: 20 January 2017

Aquaporins (AQPs) are membrane proteins that are mainly known as water channels for water transportation; however, in some cases small molecules such as glycerol, urea and ammonia can also be transported across plasma membranes through these channels [1]. Emerging evidence proposes that AQPs may also facilitate gas (e.g., carbon dioxide) and cation transport. Besides their role in the transportation of molecules, AQPs have also been implicated in the regulation of expression of other membrane proteins, cell adhesion and cell signaling [2].

Up to this point, 13 different mammalian AQPs have been identified (AQP0-12) [3]. Each member of this family is characterized with certain features and functions including tissue distribution. In recent years, AQPs have become an attractive research focus in health and disease. A range of pathophysiological conditions has been linked to certain members of AQPs that consequently highlight them as potential drug targets. Studies on genetically modified rodents and also in humans with altered aquaporin genes have provided remarkable insights into AQPs' roles [1].

Accumulating evidence supports the physiological and pathological role of $A Q P$ expression and function in the nervous system. Several AQPs are expressed in the brain and spinal cord including AQP1, AQP3, AQP4, AQP5, AQP8 and AQP9 [1]. Involvement of AQP1 and AQP4 in pain and neuroinflammation has been reported. In a rat model of spinal cord injury (SCI), a possible link between AQP1 and chronic neuropathic pain and allodynia after SCI was demonstrated [4]. AQP4-deficient mice have also been presented with reduced neuroinflammation in an experimental autoimmune encephalomyelitis, suggesting that AQP4 might contribute in neuroinflammation [5]. The roles of AQPs in the CNS have been studied more extensively. However, many questions remain unanswered about the roles of AQPs in the peripheral nervous system and in particular, in peripheral pain signaling [6-8]. A number of AQPs (AQP1, AQP2 and AQP4) have been proposed thus far to contribute in peripheral nociception, which will be presented here briefly to stimulate further research.

\section{Aquaporin1}

Small diameter sensory neurons in the dorsal root, trigeminal and nodose ganglia express AQP1. Glial cells of the peripheral nervous system also express AQP1 [9]. Injured Schwann cells in the peripheral nervous system are also known to express AQP1 [10]. Conflicting results have been

\section{KEYWORDS}

- aquaporins $\bullet$ CNS • inflammatory - neuropathic $\bullet$ nociception $\bullet$ pain - peripheral nervous system 
“...ongoing research efforts is of great importance to further understand the precise role of aquaporin family members and if selective aquaporin-based therapeutics can be used in humans for future treatment of pain." presented for a possible role for AQP1 in nociception. Using AQP1-deficient animals, it has been shown that AQP1 is involved in inflammatory thermal pain [11]. In $A Q P 1$-knockout mice, osmotically induced swelling of the spinal cord was reduced and pain sensation in response to thermal and chemical stimuli was impaired. These observations suggest a role for AQP1 in both water recycling and neural signal transduction in inflammatory thermal pain. The exact mechanism underlying this finding is not known, but an impaired physical and functional interaction between AQP1 expression and Nav1.8 function in dorsal root ganglion neurons has been proposed [12]. While based on these studies it is proposed that AQP1deficient mice are less sensitive to certain noxious stimuli including inflammatory thermal pain, Shields et al. [13] reported that AQP1-deficient mice do not have an altered nociception. This group tested behavioral responses of wild-type and AQP1-deficient mice in both acute and persistent pain models [13]. The discrepancy between findings could be due to the different experimental models used or the de novo expression of other AQPs [14]. Hence, the role of AQP1 in nociception remains to be determined.

Mutations in AQP1 occur naturally and in rare cases it has been reported in humans, that result in lack of functional AQP1 [15]. Those patients exhibit an impaired ability to produce concentrated urine under water deprivation conditions and a decreased pulmonary vascular permeability in response to fluid challenge. However, $A Q P 1$-deficient individuals do not show any neurological dysfunction [15] and no evidence is available to present any altered pain sensation in this population.

In a model of experimental temporomandibular joint osteoarthritis in rats, $\mathrm{AQP1}$ and $\mathrm{AQP} 3$ expressions under normal and osteoarthritic conditions were evaluated [16]. AQP3, but not AQP1, was highly expressed in the osteoarthritic cartilage and was considered a player in the pathogenesis of osteoarthritis [16]. It is currently unknown whether AQP3 would play any role in nociception associated with osteoarthritis.

A group of researchers speculated that AQP1 might contribute to the pathogenesis of migraine [17]. This group found that in wildtype mice, epidural application of $\mathrm{KCl}$ that can evoke cortical spreading depression induced higher expression of AQP1 in upper cervical dorsal horn neurons, but not within trigeminal ganglion in the periphery [17], which was still considered a novel finding in relation to pathogenesis of migraine. Silencing of AQP1 gene in mice using viral RNA interference method can diminish elevated light avoidance reaction in animal models of photophobia, which is often observed in migraine patients $[17,18]$.

\section{Aquaporin2}

Expression and role of AQP2 in peripheral pain transmission have also been investigated. In a perioral inflammatory pain model induced by formalin injection in mice, an altered expression of AQP2 was found in the trigeminal ganglia [19]. AQP2 expression was higher in small-size neurons and lower in neurons with medium and large size. In this model, quantity and pattern of AQP1 expression in the ganglia remained unchanged. Findings from this study highlighted a potential role of AQP2 in nociception transmission and that AQP upregulation might be specific to a certain disease or a particular pain condition [19].

Expression of AQP2 was also investigated in a model of neuropathic pain by constriction injury to sciatic nerve. In control rats, AQP2 expression was not detectable in the dorsal root ganglia; however, AQP2 expression was elevated in small-diameter neurons in response to injury [14]. These findings indicated that AQP2 is involved in neuropathic pain. Further studies are required to clarify mechanisms underlying elevated expression of AQP2 within sensory ganglia and also reasons for re-distribution of this channel in the neuronal membrane observed in these two models.

Mutations in the $A Q P 2$ gene cause hereditary nephrogenic diabetes insipidus in humans [20]. No evidence is available to present any altered pain sensation in this population.

\section{Aquaporin 4}

AQP4 is extensively expressed in astrocytes within CNS. AQP4 plays a major role in water homeostasis and impairment in its function has been linked to pathological conditions and disturbances in neuronal activity [9]. For example, pathogenesis in various neuroinflammatory diseases such as neuromyelitis optica (NMO), multiple sclerosis and brain injury has been linked to AQP4 [21]. NMO is a rare disorder that affects optic nerve and spinal cord. Anti-AQP4 is a feature of NMO and is now widely used to diagnose this disorder from multiple sclerosis. 
Pain is highly prevalent in NMO patients most commonly located around the chest and waist, along the entire length of the legs, or in the back. The mechanism underlying pain in NMO is not fully understood as of yet, however it has been proposed to arise - at least in part - directly from the development of lesions in several structures in the CNS containing or close to nociceptive pathways [22].

AQP4 upregulation in astrocytes has been suggested to contribute to the development of chronic pain [23]. One of the potential mechanisms linking AQP4 to pain processing has been proposed to involve excess glutamate release from astrocytes causing hyperexcitability in neurons [24]. In a recent study, peripheral nerve injury was shown to be associated with a longlasting AQP4 upregulation and enlargement of astrocytic processes in the rat spinal cord [25]. AQP4 expression was investigated in a rat model of SCI and mRNA levels of glial fibrillary acidic protein and AQP4 were shown to be significantly increased in the spinal cord [23]. However, in a rat model of lumbar radiculopathy, no alteration of AQP4 expression was observed between control and treated animals [26]. Investigation of a potential link between AQP4 and opioid analgesia has indicated that AQP4 deficiency could potentiate morphine analgesia, and attenuate morphine tolerance and physical dependence [27]. One study so far has tested the hypothesis of different $\mathrm{AQP} 4$ genotypes in association with migraine, however no susceptibility factor has been found [28].

Little is known about the expression or function of AQP4 in the peripheral nervous system. AQP4 expression has been shown in the myenteric and submucosal nerve plexuses in the colon of both mice and rats [29]. Interestingly, glial cells in the enteric plexuses did not express AQP4. This finding highlights differential expression of AQP4 in peripheral versus CNS. Co-localization of AQP4 with markers of nociception was not analyzed and therefore it was not clear if AQP4 is also expressed in sensory neurons of enteric system [29]. AQP4 expression in peripheral sensory ganglia was reported by Kato et al. [30] who identified that AQP4 is expressed in sensory ganglia, both in trigeminal ganglia and dorsal root ganglia. AQP4 is expressed in satellite glial cells with expression levels lower than astrocytes. Behavioral tests of mechanical and cold sensitivity between control and AQP4deficient mice did not show any significant difference. Hence, the potential role of AQP4 in peripheral nociception remains open for further investigation.

\section{Future perspective}

Ongoing research will further clarify physiological and pathophysiological roles of AQPs in nociception and pain both in animals and humans. Clarification of the involvement of AQPs in the nervous system will also drive new methods for differential diagnosis. An important direction of research is represented by studies leading to new methods for diagnosis and therapy of diseases based on progress in understanding cellular localization and regulation in expression of AQPs. Modulation of AQP expression or function could provide novel drugs or therapies for many disorders including chronic pain. In particular, investigation of local functional relationship between AQP expression and certain anatomical circuits could offer new insight into the diverse roles of AQPs in peripheral versus central mechanisms involved in perception of pain. In this line, it is highly valuable to identify and characterize AQPs of neuronal and glial cells involved in pain signaling, especially in glial cells during different glial cell functions, such as differentiation, metabolism and excitability of neurons. Identification of disease-related aquaporin polymorphisms is also advancing but at present, association of polymorphism in AQPs with certain pain conditions (e.g., migraine) is lacking. If a correlation exists between expression of AQPs and development of chronic pain, identification of risk factors and preventive strategies would be highly beneficial. Potential also exists in targeting AQPs that are overexpressed in pain pathways, and hence, development of potent and selective modulators, in particular inhibitors, is highly attractive. However, suitable monoclonal antibodies, small molecule inhibitors or AQP gene transfer with acceptable efficacy and safety profiles are still lacking. This has been due to difficulties establishing standard and reliable assays with AQP-dependent reduction of water flux. The role of AQPs in nociception is only beginning to be elucidated, therefore, ongoing research efforts is of great importance to further understand the precise role of AQP family members and if selective AQP-based therapeutics can be used in humans for future treatment of pain. The multifunctional role of AQPs combined with translation of basic research into the clinic and "...it is highly valuable to identify and characterize aquaporins of neuronal and glial cells involved in pain signaling, especially in glial cells during different glial cell functions, such as differentiation, metabolism and excitability of neurons." 
vice versa would facilitate future targeting and modulation of AQPs in both inflammatory and neuropathic pain conditions.

Financial \& competing interests disclosure

The author has no relevant affiliations or financial involve-

ment with any organization or entity with a financial interest in or financial conflict with the subject matter or materials discussed in the manuscript. This includes employment, consultancies, honoraria, stock ownership or options, expert testimony, grants or patents received or pending, or royalties.

No writing assistance was utilized in the production of this manuscript.

\section{References}

1 Papadopoulos MC, Verkman AS. Aquaporin water channels in the nervous system. Nat. Rev. Neurosci. 14(4), 265-277 (2013).

2 Kitchen P, Day RE, Salman MM, Conner MT, Bill RM, Conner AC. Beyond water homeostasis: diverse functional roles of mammalian aquaporins. Biochim. Biophys. Acta. 1850(12), 2410-2421 (2015).

3 Benga G. On the definition, nomenclature and classification of water channel proteins (aquaporins and relatives). Mol. Aspects Med. 33(5-6), 514-517 (2012).

4 Nesic O, Lee J, Unabia GC et al. Aquaporin 1 - a novel player in spinal cord injury. J. Neurochem. 105(3), 628-640 (2008).

5 Li L, Zhang H, Varrin-Doyer M, Zamvil SS, Verkman AS. Proinflammatory role of aquaporin- 4 in autoimmune neuroinflammation. FASEB J. 25(5), 1556-1566 (2011).

6 Borsani E. Aquaporins in sensory and pain transmission. Curr. Neuropharmacol. 8(2), 122-127 (2010).

7 Ma TH, Gao HW, Fang XD, Yang H. Expression and function of aquaporins in peripheral nervous system. Acta Pharmacol. Sin. 32(6), 711-715 (2011).

8 Ma T, Gao H, Fang X, Yang H. Water channel proteins in the peripheral nervous system in health and disease. Mol. Aspects Med. 33(5-6), 605-611 (2012).

9 Albertini R, Bianchi R. Aquaporins and glia. Curr. Neuropharmacol. 8(2), 84-91 (2010).

10 Gao H, He C, Fang X et al. Localization of aquaporin-1 water channel in glial cells of the human peripheral nervous system. Glia. 53(7), 783-787 (2006).

11 Oshio K, Watanabe H, Yan D, Verkman AS, Manley GT. Impaired pain sensation in mice lacking aquaporin-1 water channels. Biochem.
Biophys. Res. Commun. 341(4), 1022-1028 (2006).

12 Zhang H, Verkman AS. Aquaporin-1 tunes pain perception by interaction with $\mathrm{Na}(\mathrm{v}) 1.8$ $\mathrm{Na}^{+}$channels in dorsal root ganglion neurons. J. Biol. Chem. 285(8), 5896-5906 (2010).

13 Shields SD, Mazario J, Skinner K, Basbaum AI. Anatomical and functional analysis of aquaporin 1 , a water channel in primary afferent neurons. Pain 131(1-2), 8-20 (2007).

14 Buffoli B, Borsani E, Rezzani R, Rodella LF. Chronic constriction injury induces aquaporin-2 expression in the dorsal root ganglia of rats. J. Anat. 215(5), 498-505 (2009).

15 Preston GM, Smith BL, Zeidel ML, Moulds JJ, Agre P. Mutations in aquaporin-1; in phenotypically normal humans without functional CHIP water channels. Science 265(5178), 1585-1587 (1994).

16 Meng JH, Ma XC, Li ZM, Wu DC. Aquaporin-1 and aquaporin-3 expressions in the temporo-mandibular joint condylar cartilage after an experimentally induced osteoarthritis. Chin. Med. J. (Engl.) 120(24), 2191-2194 (2007).

17 Xu GY, Wang F, Jiang X, Tao J. Aquaporin 1, a potential therapeutic target for migraine with aura. Mol. Pain. 6, 68 (2010).

18 Wakayama Y, Inoue M, Takahashi J. Aquaporin 1 may be involved in the pathophysiology of migraine: a hypothesis. Headache 47(10), 1457-1458 (2007).

19 Borsani E, Bernardi S, Albertini R, Rezzani $\mathrm{R}$, Rodella LF. Alterations of $A Q P 2$ expression in trigeminal ganglia in a murine inflammation model. Neurosci. Lett. 449(3), 183-188 (2009).

20 Bockenhauer D, Bichet DG. Pathophysiology, diagnosis and management of nephrogenic diabetes insipidus. Nat. Rev. Nephrol. 11(10), 576-588 (2015).
21 Lan YL, Fang DY, Zhao J, Ma TH, Li S. A research update on the potential roles of aquaporin 4 in neuroinflammation. Acta Neurol. Belg. 116(2), 127-134 (2016).

22 Bradl M, Kanamori Y, Nakashima I et al. Pain in neuromyelitis optica - prevalence, pathogenesis and therapy. Nat. Rev. Neurol. 10(9), 529-536 (2014).

23 Nesic O, Guest JD, Zivadinovic D et al. Aquaporins in spinal cord injury: the janus face of aquaporin 4. Neuroscienc. 168(4), 1019-1035 (2010).

24 Mulligan SJ, Macvicar BA. VRACs CARVe a path for novel mechanisms of communication in the CNS. Sci. STKE 2006(357), pe 42 (2006).

25 Oklinski MK, Choi HJ, Kwon TH. Peripheral nerve injury induces aquaporin- 4 expression and astrocytic enlargement in spinal cord. Neuroscience 311, 138-152 (2015).

26 Lacroix-Fralish ML, Tawfik VL, Tanga FY, Spratt KF, Deleo JA. Differential spinal cord gene expression in rodent models of radicular and neuropathic pain. Anesthesiology 104(6), 1283-1292 (2006).

27 Wu N, Lu XQ, Yan HT et al. Aquaporin 4 deficiency modulates morphine pharmacological actions. Neurosci. Lett. 448(2), 221-225 (2008).

28 Rubino E, Rainero I, Vaula G et al. Investigating the genetic role of aquaporin 4 gene in migraine. J. Headache Pain 10(2), 111-114 (2009).

29 Thi MM, Spray DC, Hanani M. Aquaporin-4 water channels in enteric neurons. J. Neurosci. Res. 86(2), 448-456 (2008).

30 Kato J, Takai Y, Hayashi MK et al. Expression and localization of aquaporin- 4 in sensory ganglia. Biochem. Biophys. Res. Commun. 451(4), 562-567 (2014). 